\title{
Trabecular Bone Score, Bone Mineral Density and Bone Markers in Patients with Primary Hyperparathyroidism 2 Years After Parathyroidectomy
}

Authors

Gonzalo Allo Miguel' , Federico Hawkins Carranza ${ }^{1}$, Juan Carlos Romero Rodríguez ${ }^{1}$, Mercedes Aramendi Ramos², David Lora Pablos ${ }^{3}$, Eduardo Ferrero Herrero ${ }^{4}$, Guillermo Martínez Díaz-Guerra1

Affiliations

1 Endocrinology and Nutrition Department, 12 de Octubre University Hospital, Madrid, Spain

2 Laboratory Department, 12 de Octubre University Hospital, Madrid, Spain

3 Clinical Research Unit, IMAS12-CIBERESP, Hospital Universitario 12 Octubre, Madrid, Spain

4 General Surgery Department, 12 de Octubre University Hospital, Madrid, Spain

Key words

trabecular bone score, hyperparathyroidism, bone mineral density

received $\quad 10.06 .2018$

accepted $\quad 30.01 .2019$

Bibliography

DOI https://doi.org/10.1055/a-0850-8679

Horm Metab Res 2019; 51: 186-190

(c) Georg Thieme Verlag KG Stuttgart · New York

ISSN 0018-5043

Correspondence

Gonzalo Allo Miguel

Endocrinology and Nutrition Department

12 de Octubre University Hospital

Avenida de Córdoba S/N

28042 Madrid

Spain

Tel.: + 34/91/3908 000, Fax: + 34/91/3908 000

gonzaloallo.endo@gmail.com

\section{ABSTRACT}

Following a parathyroidectomy there is a bone mineral density (BMD) improvement in patients with primary hyperparathyroidism. However, data of bone microarchitecture are scarce. Trabecular bone score (TBS) estimates bone microarchitecture and could provide valuable information in those patients. The aim of this study is to assess TBS changes 2 years after successful surgery in a group of patients with primary hyperparathyroidism and correlate these results with changes in BMD and bone turnover markers. This is a prospective study including 32 patients. In all participants BMD and TBS were measured, before and 24 months after surgery. Biochemical data: serum calcium, PTH, 25-OH-vitamin D, beta-crosslaps, bone alkaline phosphatase, and osteocalcin. 25 female and 7 male patients, mean age $64.6 \pm 12.4$ years, were included in the study. At baseline, BMD was low at: lumbar spine (T-score $-2.19 \pm 1.31$ ), total hip $(-1.33 \pm 1.12)$, femoral neck $(-1.75 \pm 0.84)$, and distal one-third radius $(-2.74 \pm 1.68)$. Baseline TBS showed partially degraded microarchitecture $(1.180 \pm 0.130)$. After parathyroidectomy lumbar spine BMD increased significantly $(5.3 \pm 13.0 \%, \mathrm{p}<0.05)$, as well as total hip $(3.8 \pm 8.8 \%, \mathrm{p}<0.05)$. There was an increase in TBS, but this was not significant. There was a correlation between TBS and BAP at baseline $\left(r_{s}=0.73 ; p<0.01\right)$ and TBS and BAP 2 years after surgery $\left(r_{s}=0.57, p<0.05\right)$. Although bone density improves 2 years after surgery in patients with primary hyperparathyroidism and there is a restoration of bone turnover markers, TBS is not completely restored. These results remark the necessity of longer periods of study, to confirm if bone microarchitecture could be completely restored after surgery.

\section{Introduction}

Bone disease in primary hyperparathyroidism (PHPT) is characterized by bone mineral density (BMD) reduction at cortical sites (onethird distal radius, $1 / 3 \mathrm{DR}$ ) with a relative preservation of the lumbar spine (LS, predominantly a trabecular site), as assessed by dual-energy X-ray absorptiometry (DXA) [1,2]. In spite of this, not only the risk of non-vertebral fracture is increased in PHTP, but also the risk of vertebral fractures $[3,4]$. Thus, DXA may not give com- plete information about other factors that could increase the risk of fracture such as bone microarchitecture. Other techniques for skeletal evaluation, such as trabecular bone score (TBS), could provide information on trabecular bone involvement in PHPT, and eventually might contribute to redefine surgical criteria.

TBS is a reproducible and non-invasive technique that evaluates pixel gray-level variations in DXA image [5]. An elevated TBS appears to represent strong, fracture-resistant microarchitecture, 
while a low TBS reflects a heterogeneous and more porous trabecular network and reduced bone strength [6]. Previous studies using TBS have found that cortical and trabecular skeletal compartments are affected in patients with PHPT. Romagnoli et al. reported that in a group of postmenopausal women, TBS was lower in patients with PHPT than in age-matched controls [7]. Also, lower TBS values associated with vertebral fractures has been recently described in patients with PHPT [8].

Following a parathyroidectomy (PTX) (an effective therapy that cures PTHP) there is an improvement in BMD [9]. However, data of other bone determinants such as microarchitecture are scarce [10]. Thus, the aim of our study is to assess TBS changes 2 years after successful parathyroidectomy in PTHP patients and compare this result with changes observed in BMD and bone turnover markers.

\section{Patients and Methods}

\section{Study design}

This prospective study was performed in a single center (12 de Octubre University Hospital, Madrid, Spain), in the Mineral Metabolism Unit, from 2010-2015. The Ethics Committee of our Hospital approved the study and informed consent was obtained from all patients. They were evaluated at baseline (before the procedure) and 2 years after the PTX. In both visits, DXA scans and fasting blood samples were obtained. TBS was assessed from previous DXA spine exams.

All procedures performed in studies involving human participants were in accordance with the ethical standards of the institutional and/or national research committee and with the $1964 \mathrm{Hel}-$ sinki declaration and its later amendments or comparable ethical standards. Informed consent was obtained from all individual participants included in the study.

\section{Patients}

A total of 32 Caucasian patients with PHPT (25 females, 7 males; mean age $64.6 \pm 12.4$ ) voluntarily agreed to participate in the study and signed the consent form. The female group included 21 postmenopausal women. Nine patients were symptomatic. Diagnosis of PHPT was based in high calcium level and non-suppressed PTH level, with no evidence of secondary hyperparathyroidism. Familial hypocalciuric hypercalcemia was excluded (calcium/creatinine clearance ratio < 0.01 ) in every patient. Exclusion criteria were: patients with diseases known to affect bone metabolism, such as: chronic renal failure, thyrotoxicosis or rheumatological diseases; or patients who were being treated with medications that could alter bone metabolism (glucocorticoids, bisphosphonates, or denosumab). After the procedure, parathyroid adenoma and/or hyperplasia were histologically confirmed in all the patients. PTH and calcium level of every patient normalized after surgery.

\section{Bone mineral density and trabecular bone score}

BMD was assessed by DXA, (densitometer QDR 4500, Hologic, Waltham, MA, USA) at LS, femoral neck (FN), total hip (TH) and 1/3DR, with a precision error $\leq 1.5 \%$ at all sites. BMD values are expressed as absolute values $\left(\mathrm{g} / \mathrm{cm}^{2}\right)$ and T-score. According to the WHO criteria, patients were classified as: osteoporotic (T-score $\leq-2.5$ ), osteopenic $(-1 \geq$ T-score $>-2.5)$, and normal (T-score $>-1)[11]$.
TBS measurements were performed retrospectively applying the TBS iNsight2.0 software (Med-Imaps, Swiss, Switzerland) to the LS DXA exams. Lumbar TBS was calculated as the mean value of individual measurements for vertebrae $\mathrm{L} 1-\mathrm{L} 4$. Reference values: TBS $\geq 1.350$ is considered normal; TBS between 1.350 and 1.200 is consistent with partially degraded microarchitecture, and TBS $\leq 1.200$ with degraded microarchitecture $[5,6]$.

\section{Biochemical analysis}

Serum samples for biochemical analyses were obtained between 8:00 and 9:00 AM after overnight fast and immediately kept frozen at $-70^{\circ} \mathrm{C}$. Serum calcium levels (normal values: $8.4-10.2 \mathrm{mg} / \mathrm{dl}$ ) were measured by autoanalyzer (Modular P; Roche Diagnostic). Corrected calcium level was based on the serum albumin level. Urinary calcium was measured in 24-h urine collections. Serum bone markers included: Osteocalcin: OC (N-MID Osteocalcin, Roche Diagnostics, electrochemiluminescence, normal range: $8-48 \mathrm{ng} / \mathrm{ml}$ ), bone alkaline phosphatase: BAP (IDS EIA, normal range 4.0$20.0 \mathrm{ng} / \mathrm{ml}$ ) and $\beta$-crosslaps: $\beta$-CTX (ELECSYS 1010, Roche, electrochemiluminescence, normal range $0.200-0.704 \mathrm{ng} / \mathrm{ml}$ ). Serum intact PTH was determined using chemiluminescent immunometric assay (IMMULITE 2000, DPC, normal range 7.0-57.0 pg/ml). Serum 25-hydroxyvitamin D3 [25(OH)D] was measured by enzyme immunoassay (automated IDS EIA, kit). Vitamin D deficiency was defined as a $25(\mathrm{OH}) \mathrm{D}$ level below $20 \mathrm{ng} / \mathrm{ml}$, insufficiency as a value between $>20$ and $<30 \mathrm{ng} / \mathrm{ml}$, whilst a $25(\mathrm{OH})$ D value $>30 \mathrm{ng} / \mathrm{ml}$ could indicate vitamin D sufficiency [12].

\section{Statistical analysis}

Normality was assessed using the Shapiro-Wilk test. Continuous variables are described as mean and standard deviation and categorical variables as percentage. Changes in parameters compared with baseline (e. g., BMD and TBS) were analyzed using Wilcoxon's test. Correlation analysis between continuous parameters was performed by Spearman's test. Statistical analyses were conducted using SAS software (version 9). Significance level assumed was $\mathrm{p}<0.05$.

- Table 1 Biochemical values of PHPT patients $(n=32)$ at baseline and after 2 years of follow-up.

\begin{tabular}{|l|c|c|c|}
\hline & Baseline & 2 Years & p-Value \\
\hline $25(\mathrm{OH}) \mathrm{D}(\mathrm{ng} / \mathrm{ml})$ & $22.9 \pm 12.3$ & $26.5 \pm 10.5$ & n.s. \\
\hline PTH $(\mathrm{pg} / \mathrm{ml})$ & $171.7 \pm 70.6$ & $50.1 \pm 19.65$ & $<0.001$ \\
\hline $\begin{array}{l}\text { Serum corrected } \\
\text { calcium }(\mathrm{mg} / \mathrm{dl})\end{array}$ & $11.2 \pm 0.7$ & $9.5 \pm 0.4$ & $<0.001$ \\
\hline $\begin{array}{l}\text { Urinary calcium in } \\
24 \mathrm{~h}(\mathrm{mg} / 24 \mathrm{~h})\end{array}$ & $392.8 \pm 252.3$ & $165.9 \pm 132.1$ & $<0.01$ \\
\hline \begin{tabular}{l} 
B-CTX $(\mathrm{ng} / \mathrm{ml})$ \\
\hline Osteocalcin $(\mathrm{ng} / \mathrm{ml})$
\end{tabular} & $47.25 \pm 17.46$ & $17.86 \pm 7.99$ & $<0.05$ \\
\hline BAP $(\mathrm{ng} / \mathrm{ml})$ & $50.96 \pm 36.98$ & $27.39 \pm 17.42$ & n.s. \\
\hline
\end{tabular}




\section{Results}

Data were collected from 32 Caucasian patients, 25 females (78.1\%) and 7 males (21.9\%). Mean age at diagnosis was 64.6 \pm 12.4 years. Mean BMI at baseline was $29.1 \pm 5.1 \mathrm{~kg} / \mathrm{m}^{2}$. Mean time between diagnosis and surgery was less than 1 year. $>$ Table 1 shows biochemical values before and after the PTX. At baseline there were no statistically significant differences in any of the laboratory values between gender groups. Low serum $25(\mathrm{OH}) \mathrm{D}$, in the range of insufficiency, was found in our PHPT patients before surgery. As expected, mean serum PTH, serum calcium and urinary calcium in $24 \mathrm{~h}$ were significantly higher at baseline, and normalized after surgery. 25(OH)D showed a non-significant improvement after 2 years, but persisted in the insufficiency range. Baseline turnover markers were above the reference range. $\beta$-CTX and OC showed a statistically significant decrease 2 years after the surgery treatment. Gender had no influence on the changes in bone turnover markers.

BMD, T-score, and TBS values are shown in $>$ Table 2 . At baseline, mean T-score values were in the osteoporotic range at 1/3DR site and in the osteopenia range at LS, TH and FN sites. Similar results were found in both genders; except for mean TH T-score in the male group, which was in the normal range. Mean LS T-score increased throughout the study, and after 2 years there was a significant increase (compared to baseline), with a percent of change of change in LS BMD of $+5.3 \% \pm 13.0(p<0.001)$. Male and female groups showed similar results, with statistically significant increases at mean LS T-score at the end of the study $(p=0.001$ and $p=0.01$, respectively).

After 2 years, mean 1/3DR T-score showed a non-significant improvement. In the female group there was a small decrease of mean 1/3DR T-score. In the total group, mean TH and FN T-scores showed non-significant increases after 2 years, and final values persisted in the osteopenia range. Similar results were found in both genders.

Baseline TBS showed degraded microarchitecture in the total and female group ( $1.18 \pm 0.13$ and $1.15 \pm 0.11$, respectively). Male group TBS value was consistent with partially degraded microarchitecture (1.28 \pm 0.13$)$. No significant correlation was found between baseline TBS and BMD values. There were no significant differences in TBS between vitamin D-deficient and vitamin D-sufficient patients. A positive correlation was found between TBS at baseline and BAP $\left(r_{s}=0.73 ; p<0.01\right)$. At the end of the study TBS showed a non-significant improvement reaching the partially degraded range in 19 patients (percent of change of $+4.0 \% \pm 12.4$ ). In the female group TBS persisted in the degraded range and in the male group in the partially degraded range ( $\vee$ Table 2 ). Final TBS showed positive statistically significant correlation with BAP after 2 years $\left(r_{s}=0.57 ; p<0.03\right)$.

\section{Discussion}

In this prospective study, we have assessed the influence of PTX in PHPT patients on BMD, TBS, and bone turnover markers. To our knowledge, this study is one of the longest and largest evaluating the effects of PTX on TBS. Baseline score showed degraded microarchitecture, predominantly in females. After 2 years, TBS increased non-significantly reaching the partially degraded range.

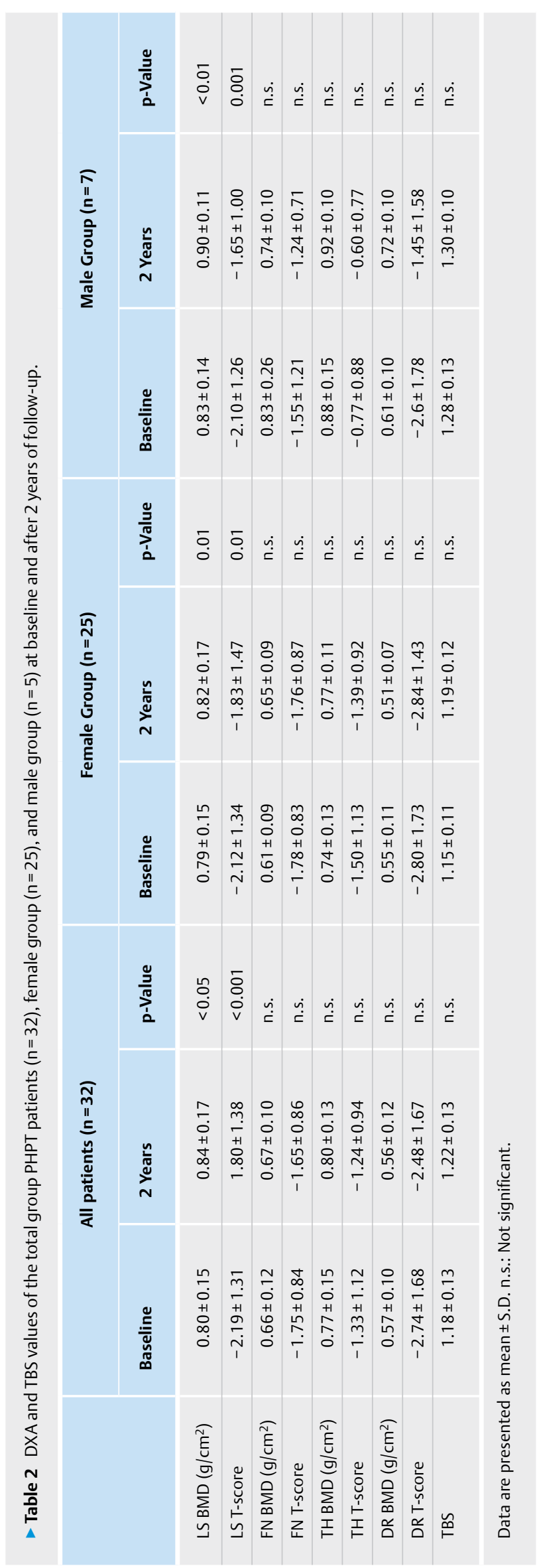


Patients with PHPT tend to have decreased BMD, in particular at cortical sites (1/3DR and hip) as compared with trabecular sites (LS) [5], because of the catabolic effects of PTH in cortical bone [13]. In agreement with this, our results showed BMD in the osteoporotic range at $1 / 3 \mathrm{DR}$ site and in the osteopenia range at $\mathrm{LS}, \mathrm{TH}$ and FN sites.

In the past years, several studies have assessed the effect of surgical treatment on BMD in PHPT patients, demonstrating increased $\mathrm{BMD}$ at $\mathrm{TH}$ and/or $\mathrm{LS}$ with high resolution peripheral quantitative computed tomography (HR-pQCT) [10]. Those increases in BMD remain significantly above baseline even in the long term [14]. Hansen et al. reported postoperative improvements at LS, 1/3DR and TH in 27 women 1 year after PTX [10] and Lundstam et al. postoperative improvements at LS, and FN after 5 years compared to observation [15]. Similarly, our results showed significant increase of LS BMD and non-significant increases of 1/3DR, TH, and FN. Surprisingly female patients showed non-significant decrease of $1 / 3 D R$. To our knowledge there are no previous reports of BMD decrease in women at 1/3DR after PTX.

Regarding bone microarchitecture, our results showed degraded microarchitecture at baseline $(1.18 \pm 0.13)$, considering the broadly accepted parameter of TBS $\leq 1.200$. Our results are in agreement with the cross-sectional study of Rogmanoli et al. [7] reporting that TBS was reduced in 73 postmenopausal women with PHTP (1.19 \pm 0.10$)$ compared to controls (1.24 \pm 0.09$)$. In our cohort, female patients showed lower TBS value (degraded range) than the male group (partially degraded range).

Recently Eller-Vainicher et al. [8] have published a longitudinal study evaluating the effect of surgery on TBS after 2 years of follow-up (20 patients). In the surgical group TBS increased significantly, but was still lower compared to controls. Also, Hansen et al. described a significantly improved microarchitecture after parathyroidectomy assessed by HRPQCT [10]. Conversely, in our cohort, TBS improvement did not reach statistical significance. Tay et al. [16] have recently reported similar results to ours: TBS value did not improve significantly postparathyroidectomy in either obese or nonobese subjects after 2 years, and was minimally below normal throughout the study despite improvements in BMD.

Bone markers are used to assess bone turnover and to provide information about the metabolic status of bone, but their role in the evaluation and management of PHPT is not well established [17]. Our results show that, at baseline, all turnover markers were above the reference range; probably due to the increased bone turnover characteristic of PHPT [6]. Previously, other groups have published similar results $[18,19]$. In our cohort, 2 years after PTX $O C$ and $\beta$-CTX fell significantly into the normal range, while BAP persisted elevated. Formation markers (like BAP) are supposed to decline more slowly than resorption markers [20] after PTX. This could explain the lack of significant decrease of BAP after 2 years.

BAP showed positive statistically significant correlations with TBS value at baseline and at the end of the study. There are no previous reports of this kind of correlation in PHPT, although in a previous study our group has described that baseline serum $\beta C T X$ and P1NP levels were related to lumbar BMD changes one year after PTX [21]. Future studies should try to identify if BAP could also be a specific bone formation marker that could predict beneficial effects on bone microarchitecture after PTX.
Our study did not detect significant TBS differences between groups according vitamin $D$ status. This could reflect that vitamin $D$ have an effect upon mineralization but not microarchitecture or may be related to the low sensitivity of TBS. These results are consistent with those described in 2 recent studies in PHPT patients [10,22].

The strength of this study is that it provides information not only about BMD and microarchitecture but also on bone turnover marker dynamics in PHPT patients after PTX. All PHPT patients were followed during the 2 years of the study, without dropouts. This study has some limitations as the small sample size and the lack of systematic assessment of morphometric vertebral fractures.

In conclusion, our study demonstrates that although BMD improves 2 years after surgery in PHPT patients, and there is a restoration of bone turnover markers, TBS is not completely restored. These results remark the necessity of longer periods of study, to confirm if bone microarchitecture has completely restored after surgery.

\section{Conflict of Interest}

The authors declare that they have no conflict of interest.

\section{References}

[1] Bandeira F, Cassibba S. Hyperparathyroidism and bone health. Curr Rheumatol Rep 2015; 17: 48

[2] Silverberg S], Shane E, Jacobs TP et al. A 10-year prospective study of primary hyperparathyroidism with or without parathyroid surgery. N Engl J Med 1999; 341: 1249-1255

[3] Vignali E, Viccica G, Diacinti D et al. Morphometric vertebral fractures in postmenopausal women with primary hyperparathyroidism. J Clin Endocrinol Metab 2009; 94: 2306-2312

[4] De Geronimo S, Romagnoli E, Diacinti D et al. The risk of fractures in postmenopausal women with primary hyperparathyroidism. Eur ] Endocrinol 2006; 155: 415-420

[5] Silva BC, Boutroy S, Zhang C et al. Trabecular bone score (TBS) - A novel method to evaluate bone microarchitectural texture in patients with primary hyperparathyroidism. J Clin Endocrinol Metab 2013; 98: 1963-1970

[6] Silverberg S], Clarke BL, Peacock M et al. Current issues in the presentation of asymptomatic primary hyperparathyroidism: Proceedings of the Fourth International Workshop. J Clin Endocrinol Metab 2014; 99: 3580-3594

[7] Romagnoli E, Cipriani C, Nofroni I et al. "Trabecular Bone Score" (TBS): An indirect measure of bone micro-architecture in postmenopausal patients with primary hyperparathyroidism. Bone 2013; 53: 154-159

[8] Eller-Vainicher C, Filopanti M, Palmieri S et al. Bone quality, as measured by trabecular bone score, in patients with primary hyperparathyroidism. Eur J Endocrinol 2013; 169: 155-162

[9] Sankaran S, Gamble G, Bolland M et al. Skeletal effects of interventions in mild primary hyperparathyroidism: A meta-analysis. J Clin Endocrinol Metab 2010; 95: 1653-1662

[10] Hansen S, Hauge EM, Rasmussen L et al. Parathyroidectomy improves bone geometry and microarchitecture in female patients with primary hyperparathyroidism: A one-year prospective controlled study using high-resolution peripheral quantitative computed tomography. J Bone Miner Res 2012; 27: 1150-1158

[11] World Health Organization. Scientific Group Research on Menopause. WHO Technical Service Report Series 670. WHO; Geneva: 1998 
[12] Holick MF. Vitamin D deficiency. N Engl J Med 2007; 357: 266-281

[13] Bilezikian JP, Brandi ML, Eastell $R$ et al. Guidelines for the management of asymptomatic primary hyperparathyroidism: Summary statement from the Fourth International Workshop. J Clin Endocrinol Metab 2014; 99: 3561-3569

[14] Lee JH, Kim JH, Hong AR et al. Skeletal effects of vitamin D deficiency among patients with primary hyperparathyroidism. Osteoporos Int 2017; 28: 1667-1674

[15] Lundstam K, Heck A, Mollerup C et al. Effects of parathyroidectomy versus observation on the development of vertebral fractures in mild primary hyperparathyroidism. J Clin Endocrinol Metab 2015; 100: 1359-1367

[16] Tay YD, Cusano NE, Rubin MR et al. Trabecular bone score in obese and nonobese subjects with primary hyperparathyroidism before and after parathyroidectomy. J Clin Endocrinol Metab 2018; 103: 1512-1521

[17] Costa AG, Bilezikian JP. Bone turnover markers in primary hyperparathyroidism. J Clin Densitom 2013; 16: 22-27
[18] Guo CY, Thomas WE, al-Dehaimi AW et al. Longitudinal changes in bone mineral density and bone turnover in postmenopausal women with primary hyperparathyroidism. J Clin Endocrinol Metab 1996; 81: 3487-3491

[19] Christiansen P, Steiniche T, Brixen K et al. Primary hyperparathyroidism: Biochemical markers and bone mineral density at multiple skeletal sites in Danish patients. Bone 1997; 21: 93-99

[20] Christiansen P, Steiniche T, Brixen K et al. Primary hyperparathyroidism: Short-term changes in bone remodeling and bone mineral density following parathyroidectomy. Bone 1999; 25: 237-244

[21] Alonso S, Ferrero E, Donat M et al. The usefulness of high pre-operative levels of serum type I collagen bone markers for the prediction of changes in bone mineral density after parathyroidectomy. J Endocrinol Invest 2012; 35: 640-644

[22] Walker MD, Saeed I, Lee JA et al. Effect of concomitant vitamin D deficiency or insufficiency on lumbar spine volumetric bone mineral density and trabecular bone score in primary hyperparathyroidism. Osteoporos Int 2016; 27: 3063-3071 